\title{
How the unit cell surface charge distribution affects the energetics of ion-solvent interactions in simulations
}

\author{
James E. Roberts and Jurgen Schnitker \\ Department of Chemistry, University of Michigan, Ann Arbor, Michigan 48109-1055
}

(Received 4 April 1994; accepted 31 May 1994)

\begin{abstract}
The evaluation of the electrostatic potential in condensed phase simulations normally includes an "extrinsic" contribution that manifests natural imbalances in the surface charge distribution of the microscopic unit cell. Most directly affected are ion-solvent interaction energies, and depending on whether the specific simulation conditions eliminate the extrinsic potential or not, these energies can vary by a considerable amount. This is illustrated by examining simulations of dilute aqueous solutions of $\mathrm{Cl}^{-}$and $\mathrm{Fe}^{2+}$ that use either a cutoff scheme or Ewald summation. It is found that the ion-water potential energy can vary with the type of boundary condition by as much as $\approx 60$ $\mathrm{kJ} \mathrm{mol}^{-1}$ for $\mathrm{Cl}^{-}$and $\approx 800 \mathrm{~kJ} \mathrm{~mol}^{-1}$ for $\mathrm{Fe}^{2+}$. The difference is exclusively due to the extrinsic potential effect and it is easy to calculate an appropriate correction term.
\end{abstract}

\section{INTRODUCTION}

There is a peculiar small system effect that regularly arises in computer simulations of ionic solutions but that has apparently not been addressed in the rather extensive literature on boundary conditions and long-range forces. ${ }^{1-3}$ The effect can give rise to a major difference between the ionsolvent energy that is calculated in a "cutoff" simulation as compared to that which is obtained in a simulation that uses Ewald summation. As this energy is usually the main contributor to the experimentally accessible ionic solvation energy it is clear that the effect can have important ramifications for the assessment of simulation data.

Interestingly, the effect is not related to the fact that the range of the potential is finite and fairly short-ranged under cutoff conditions while it is formally infinite in the case of Ewald summation. Rather it is related to a kind of surface effect that is sometimes encountered in solid-state physics. There we know, for example, that the work function for an electron, even deep inside a solid, can depend on the surface structure, ${ }^{4}$ and we also know that the evaluation of the Madelung constant requires special procedures (like Evjen's method or Ewald summation) because of difficulties associated with the surface of an infinitely replicated crystal. ${ }^{4}$ At the root of either problem is the well-known conditional convergence of Coulomb lattice sums, reflecting the pronounced long-range character of the $1 / r$ potential. In the following, we will see that the concept of surface effects generalizes beyond the case of infinite lattice sums and proves to be a sensible tool for the rationalization of simulation results of liquids and solutions.

What we are interested in is the electrostatic potential anywhere in the replicated microscopic unit cell that is typical for a condensed phase simulation. This is most easily approached by separating the total potential into an "intrinsic" and an "extrinsic" contribution. " As shown by Redlack and Grindlay in two groundbreaking papers, ${ }^{5}$ this separation is unique in the sense that the intrinsic potential has a welldefined value for any point of the unit cell and that it is a periodic function with respect to the unit cell replication. Physically we can say that the intrinsic potential represents the electrostatics of the local surroundings of a given point and the inherent structure of the medium.

The extrinsic potential, on the contrary, is related to the surface charge distribution of the replicated system. ${ }^{5-8}$ In a simulation the surface will be given by either the unit cell itself (under minimum image boundary conditions) or the truncation sphere (if a spherical cutoff is used) or the infinitely replicated unit cell (if lattice sums are evaluated). Thus the extrinsic potential is dependent on shape, size, and mode of replication of the unit cell, whereas the intrinsic potential is independent of these factors. The extrinsic potential does have real physical meaning in microscopic clusters and also in perfect macroscopic crystals. ${ }^{7,9}$ However, in a disordered system such as any liquid there is no distinct surface structure and the extrinsic potential is expected to vanish. Hence, in a liquid state simulation we are (ideally) interested in the intrinsic potential only.

There are two ways to remove the influence of the extrinsic potential. One approach, for which Evjen's method of lattice summation ${ }^{10}$ is the most famous example, involves a very special and system-specific choice of unit cell, clearly not an option for a disordered system. The other possibility is Ewald summation ${ }^{11}$ and some other methods related to it. As a purely mathematical trick the latter approach has of course been known for many decades, but it is only since the work of De Leeuw et al. ${ }^{12}$ and Felderhof ${ }^{13}$ that we know what is actually happening: the energy expression is effectively that of an infinitely replicated simulation cell that is surrounded by a fictitious medium of infinite dielectric constant. This is sometimes described as "conducting" or "tin foil" boundary conditions, and it is this choice of the macroscopic surroundings that effectively leads to a screening of all surface effects. A similar feat is accomplished in reaction field simulations (even though this is usually not given as the primary rationale for the use of this technique).

The important point is that the macroscopic surroundings can be quite different under different kinds of boundary conditions. Mathematically this translates into presence or absence of a surface term in the Hamiltonian, as worked out in detail by De Leeuw et al. ${ }^{12}$

It is well known that the latter work has important 
implications for the proper calculation of dielectric properties. ${ }^{14-16}$ In the present paper, we show that there can also be prominent energetic consequences that can be directly related to the extrinsic potential. This becomes clear by considering simulations of aqueous ionic solutions of $\mathrm{Cl}^{-}$and $\mathrm{Fe}^{2+}$ under several kinds of boundary conditions, including use of an interaction cutoff and Ewald summation.

We find that the ion-water interaction energy of, for example, the doubly charged $\mathrm{Fe}^{2+}$ ion-as calculated with the two main kinds of boundary conditions - can differ by as much as $\approx 800 \mathrm{~kJ} \mathrm{~mol}^{-1}$, or about one-third of the total interaction energy itself. We also show that either energy can easily be corrected to give the other one by including (or excluding) the "surface" term. This does not seem to be very model dependent, as we make the same set of observations with three popular water models (SPC, TIP4P, and MCY).

In the following, we first analyze the electrostatics of a simulation cell under periodic boundary conditions (Sec. II). We then describe the setup of some molecular dynamics simulations of ionic aqueous solutions (Sec. III), present the simulation results (Scc. IV), and finish with the conclusions (Sec. V).

\section{THEORY}

The central formalism relevant for the electrostatics of a replicated system can be found in papers by Redlack and Grindlay ${ }^{5}$ and by De Leeuw et al. ${ }^{12}$ While the latter work is quite often referenced, even in the textbook literature, $2,17,18$ the results of Redlack and Grindlay are less known, despite their great physical significance. Both papers systematically analyze the lattice summation problem, but from rather different and complimentary viewpoints, as Redlack and Grindlay ${ }^{5}$ focus on the electrostatic potential inside a finite cluster while De Leeuw et al. ${ }^{12}$ consider the total electrostatic energy of an infinitely replicated system. Naturally, we are interested in all of these cases, i.e., both the electrostatic potential (related to the ion-solvent energy) and the total energy expression (providing the simulation Hamiltonian) in both finite (cutoff) and infinitely replicated (Ewald) systems.

In fact, considerable physical insight is gained by relating the results of both papers to each other. In the following, we will therefore (i) summarize the results of Redlack and Grindlay, ${ }^{5}$ (ii) establish the connection to the work of De Leeuw et al. ${ }^{12}$ and (iii) address some issues that specifically arise in the simulation of molecular liquids and that have not been considered before.

\section{A. Periodic boundary conditions}

Particularly in connection with the last point raised it is important to have a clear concept and clear definitions of the term "periodic boundary conditions." Unfortunately, the term is in such common use that it has rather different connotations in different situations. While the general topic is of course addressed in the standard sources, ${ }^{1,2,17-20}$ we have not been able to find a systematic categorization of the kinds of boundary conditions that are commonly encountered in simulations. We therefore provide in the Appendix of this paper a list of the somewhat subtle but nevertheless important points to be considered.

\section{B. Electrostatic potential}

Following Redlack and Grindlay, ${ }^{5}$ we consider an electroneutral unit cell " $C$ " of point charges that is periodically replicated within a finite region " $L$ " such that the latter contains an integer number of unit cells. The symbol $C$ stands for all the specifications of the unit cell, and $L$ stands for the shape and volume of the region.

The electrostatic potential $\phi$ at any point $\mathbf{r}$ in any one of the unit cells is then given by the sum of two contributions [we always omit factors of $\left.1 /\left(4 \pi \epsilon_{0}\right)\right]^{5,6}$

$$
\Phi(\mathbf{r}, C, L)=\Phi_{i}(\mathbf{r})+\Phi_{e}(\mathbf{r}, C, L)
$$

The intrinsic potential $\phi_{i}$ is a periodic function of the position in the unit cell. The extrinsic potential $\phi_{e}$ is not only a nonperiodic function of $\mathbf{r}$ but also a function of the choice of unit cell $C$ and a function of the specification of the region $L$.

If the region is ellipsoidal with its center at $\mathbf{r}^{0}$, Redlack and Grindlay derive the following expression for the extrinsic potential $(i, j=x, y, z): 5,21$

$$
\begin{aligned}
\Phi_{e}(\mathbf{r}, C, L)= & \frac{4 \pi}{v}\left(\sum_{i}\left(r_{i}-r_{i}^{0}\right) p_{i} \sum_{j} S_{i j}(L)\right. \\
& \left.-\frac{1}{2} \sum_{i} \sum_{j} Q_{i j} S_{i j}(L)\right) .
\end{aligned}
$$

Here $v$ is the volume of the unit cell $C$ and $p_{i}$ and $Q_{i j}$ are the components of the cell's dipole moment vector and quadrupole moment tensor, respectively, that originate from the distribution of all the $N$ charges $q_{k}$ in the cell

$$
\begin{aligned}
& p_{i}=\sum_{k=1}^{N} q_{k} r_{i_{k}}, \\
& Q_{i j}=\sum_{k=1}^{N} q_{k} r_{i_{k}} r_{j_{k}} .
\end{aligned}
$$

The tensor $S_{i j}$ characterizes the shape of the ellipsoidal region $L$; its trace is unity.

For a cubic or spherical region, the shape tensor is simply $S_{i j}=\delta_{i j} / 3$ because of complete symmetry and the trace being unity. The direct lattice sums in standard mathematical treatments correspond to "fixed-cell mode" (see the Appendix) and we obtain for the extrinsic potential

$$
\Phi_{e}(\mathbf{r}, C, L)=\frac{4 \pi}{3 v}\left(\mathbf{r}-\mathbf{r}^{0}\right) \sum_{k} q_{k} \mathbf{r}_{k}-\frac{2 \pi}{3 v} \sum_{k} q_{k} \mathbf{r}_{k}^{2} .
$$

It can be seen that there is both a dipolar and a quadrupolar contribution. While the former is position dependent, the latter is a constant for a given configuration.

In "centered-cell mode"-which corresponds to the minimum image conditions in a standard molecular dynamics simulation-we are always finding the potential at the center $\left(\mathbf{r}=\mathbf{r}^{0}\right)$ of the region $L$, and we obtain from Eqs. (2) and (4), for a cubic or spherical region

$$
\Phi_{e}(\mathbf{r}, C, L)=-\frac{2 \pi}{3 v} \sum_{k} q_{k}\left(\mathbf{r}_{k}-\mathbf{r}\right)^{2} .
$$


Hence, in this case there is only a quadrupolar term; however, the term varies from point to point.

As shown by Redlack and Grindlay, ${ }^{5}$ Ewald summation provides the intrinsic potential

$$
\Phi_{\text {Ewald }}(\mathbf{r})=\Phi_{i}(\mathbf{r}) \text {. }
$$

Thus a "polarization correction" of the Ewald potential according to Eqs. (2), (5), or (6) recovers the total potential including the extrinsic contribution. Analogously in a procedure known as "depolarization correction," 6 it is possible to obtain the intrinsic potential by explicitly subtracting the extrinsic part from the value that is obtained by, for example, direct lattice summation.

\section{Total electrostatic energy}

We now wish to consider the electrostatic energy of the entire system by summing over all pairs of charges within the unit cell $C$. From Eq. (5) we obtain-for a cubic or spherical region and in fixed-cell mode-for the energy $E_{e}$ associated with the extrinsic potential

$$
\begin{aligned}
E_{e}= & \frac{1}{2}\left(\frac{4 \pi}{3 v} \sum_{k} q_{k}\left(\mathbf{r}_{k}-\mathbf{r}^{0}\right) \sum_{l} q_{l} \mathbf{r}_{l}\right. \\
& \left.-\frac{2 \pi}{3 v} \sum_{k} q_{k} \sum_{l} q_{l} \mathbf{r}_{l}^{2}\right),
\end{aligned}
$$

where, because of the assumcd clcctroneutrality of the unit cell, only the first term needs to be retained, i.e., the term that relates back to the dipolar part of the extrinsic potential, and we finally obtain

$$
E_{e}=\frac{2 \pi}{3 v}\left(\sum_{k} q_{k} \mathbf{r}_{k}\right)^{2} .
$$

Thus the extra energy is proportional to the square of the unit cell dipole moment, as first found by De Leeuw et al. ${ }^{12}$ and Felderhof ${ }^{13}$ and elaborated on by Olives. ${ }^{22}$

In the centered-cell mode typically dealt with in simulations we obtain from Eq. (6)

$$
E_{e}=-\frac{1}{2} \frac{2 \pi}{3 v} \sum_{k} q_{k} \sum_{l} q_{l}\left(\mathbf{r}_{l}-\mathbf{r}_{k}\right)^{2}
$$

which after some rearranging and making use of the identity

$$
-\frac{\pi}{3 v} \sum_{k} \sum_{l} q_{k} q_{l}\left(\mathbf{r}_{k}^{2}+\mathbf{r}_{l}^{2}\right)=0
$$

leads to the same end result

$$
E_{e}=\frac{2 \pi}{3 v}\left(\sum_{k} q_{k} \mathbf{r}_{k}\right)^{2}
$$

which in this case, however, originated in the quadrupolar part of the extrinsic potential.

Thus the total system Hamiltonian is not affected if the unit cell does not have a dipole moment. We note, however, that there still can be absolute shifts of the potential, Eq. (5) or Eq. (6), that cancel out in the total energy expression for the electroneutral system. These absolute shifts can be im- portant if we want to compare single particle binding energies with absolute experimental values. We also note that the absolute shifts are going to vary from charge to charge, and-if we consider the ensemble of systems generated in a finite temperature simulation-also from configuration to configuration.

If the unit cell does have a dipole moment, however, the Hamiltonian is fundamentally affected, and the total energy is the sum of two contributions

$$
E=E_{i}+E_{e},
$$

where $E_{i}$ can be obtained from a simulation using Ewald simulation and where $E_{e}$ can be designated as a polarization correction to the Ewald energy, since it explicitly introduces the polarization associated with the imbalance or asymmetry of the surface charge distribution. Analogously, a depolarization correction of a direct lattice sum Hamiltonian, with the negative of the energy $E_{e}$, removes the surface effects and recovers the intrinsic Ewald value. ${ }^{6}$

There is a subtlety in reconciling the results of De Leeuw et al. ${ }^{12}$ with those of Redlack and Grindlay, ${ }^{5}$ which does not affect simulations of pure water, but which is important for ionic solutions. The expression (6) for the extrinsic potential is given in the paper of De Leeuw et al. ${ }^{12} \mathrm{We}$ note, however, that this is the extrinsic potential in centeredcell mode, while De Leeuw et al.'s mathematical treatment is based on direct lattice sums, which should have led to the fixed-cell mode potential (5). The distinction between Eqs. (5) and (6) becomes irrelevant for point dipolar interactions, such as the Stockmayer fluid investigated by De Leeuw et al., ${ }^{12}$ and also for water. However, this is not true for any (electroneutral) system with free charges, such as molten salts or ionic solutions. Under fixed-cell mode conditions, there is also the dipolar term in Eq. (5) and the positions $\mathbf{r}_{k}$ in Eq. (5) are clearly not minimum image shifted positions. The use of minimum image shifted positions and neglect of the dipolar part is in fact suggested by De Leeuw et al., but despite their otherwise rigorous derivation this is only applicable if the centered-cell mode is ad hoc enforced.

\section{Electrostatic force}

From Eq. (9) we obtain for the total electrostatic force acting on charge $k$

$$
\mathbf{F}_{k}=-\frac{4 \pi}{3 v} q_{k} \sum_{l} q_{l} \mathbf{r}_{l} .
$$

It can be seen that the force is exactly the same for all charges of a given type. If the unit cell does not have a dipole moment, there is no net force, but the absolute value of the electrostatic potential-as mentioned before in the context of the total system energy - will still be affected in the sense of a certain shift of the potential that varies from charge to charge.

\section{E. Systems with excess charges}

We now consider ions in a solvent. There are a number of possibilities for the description of an electrolyte solution. One is that the numbers of anions and cations are properly 
matched so as to obtain electroneutrality; in this case all of the previous formalism is immediately applicable. Another option, very popular for the study of "infinitely dilute" solutions, is to have just one ion in the solvent. As this is not an electroneutral system, the outlined theory is not applicable, unless it is assumed that the excess charge is canceled by a uniform compensating background of opposite charge. (Such compensating background charges are usually not explicitly specified as they only alter the total energy of the system by an additive constant.)

Assuming the presence of a compensating background charge, there are, as before, two possibilities. In fixed-cell mode, i.e., if the ion is immersed in the solvent in a fixed cell, with the compensating charge filling out this cell, there are extra terms in the Hamiltonian, for the extrinsic ionbackground and solvent-background interactions. (Since fixed-cell mode conditions preclude use of an energyconserving molecular dynamics algorithm, this has to be implemented in a Monte Carlo type scheme.) It is then seen that there is a net tendency for the ion to be at the surface of the fixed unit cell, as the quadrupole moment of the background charge with respect to the ion site is thus maximized. Naturally this is a highly artificial and undesired situation.

Alternatively, in centered-cell mode, i.e., using the equivalent of the usual minimum image convention also for the background, the background charge is always exactly centered around the ion (or solvent site), so that the associated surface term vanishes by symmetry. Hence, in this case the presence of the background charge has no net effect on the system, and it is appropriate to use Eqs. (6), (9), and (13) by simply summing over all solvent charges plus the extra ion charge, without any real violation the electroneutrality condition.

\section{F. Molecular liquids}

In the case of molecular systems, it is important to evaluate the extrinsic potential (6) under molecular minimum image conditions, i.e., under account of the irregular distortion of the unit cell ("surface corrugation") which in fact contributes to the quadrupole moment (4). Under site minimum image conditions the latter will be obtained as a much smaller value while at the same time the dipole moment (3) will be much bigger, thus affecting the Hamiltonian Eq. (9) drastically.

\section{G. Rigid body liquids}

For any partial charge type molecular model, the surface term can be split up into contributions from intermolecular and intramolecular interactions. It is important to realize that, conceptually, the intrapart is very important as its omission would imply that the calculation of the force on a given molecule ignores the molecule's own contribution to the total unit cell dipole moment, clearly an unphysical proposition.

However, most simulations of molecular liquids are carried out using a rigid body solvent model, in the case of molecular dynamics simulations typically by enforcing a number of bond and/or angle constraints after initially unconstrained time steps. In this case the intramolecular part of the surface term does not exert a net force. Hence, it is admissible and convenient to evaluate the surface term (13) only considering intermolecular pair interactions.

\section{H. Simulations}

The setup in the following simulation studies always involves the centered-cell mode and molecular minimum image conditions. We consider both toroidal (cutoff) and periodic (Ewald) boundary conditions; the surroundings can be varied by either including or excluding the surface term from Eqs. (6), (9), and (13). In encoding the surface term, neither the solvent intramolecular contribution, nor-in the case of ionic solutions-the conceptual presence of a compensating background charge needs to be explicitly considered, as these do not make a net contribution. Finally, advantage can also be taken of the fact that the surface term force (13) is the same for all charges of the same type.

\section{MOLECULAR DYNAMICS SIMULATIONS}

Room temperature simulations of dilute aqueous solutions of $\mathrm{Cl}^{-}$and $\mathrm{Fe}^{2+}$ were carried out with three different water models and four alternative kinds of boundary conditions. In each case a cubic box was used which contained 200 rigid molecules of the SPC, TIP4P, or MCY type $\mathrm{e}^{23-25}$ plus the ionic solute. To keep the comparison between the models simple, the density was always set to the experimental value of $0.997 \mathrm{~g} \mathrm{~cm}^{-3}$ and it was assumed that the partial molar volume of the ion was negligible.

For the ion-water interaction, the distributed charges of the various water models were combined with popular shortranged potential functions that for simplicity were taken to be exactly the same for either one of the water models. For the interaction of $\mathrm{Cl}^{-}$with water, a Lennard-Jones potential was chosen with frequently used parameters for the ion in TIP4P water ${ }^{26}$ For the short-ranged interaction of $\mathrm{Fe}^{2+}$ with water, we used a slightly more complicated potential function that was originally obtained in conjunction with a flexible version of the SPC model, ${ }^{27}$ and that since has been used by many investigators for $\mathrm{Fe}^{2+}$ in both flexible and rigid SPC water.

The Coulomb interactions were evaluated with either a molecular minimum image cutoff, based on the oxygen position, or using Ewald summation. In two additional simulation modes, the extrinsic potential of Eq. (6) was applied to all water-water and water-ion interactions in the sense of either a cutoff simulation with depolarization correction, or Ewald simulation with polarization correction. Ewald summation was always carried out with a direct method using fast single sums. ${ }^{19}$ In the case of cutoff conditions, a true minimum image cubic cutoff, i.e., a cutoff that includes all molecules of the unit cell, was preferred over a spherical cutoff in order to have conditions as comparable as possible, with respect to symmetry, to those in the simulations using Ewald summation. In order to have energy conservation in the cutoff simulations, the Coulomb interactions (and the correction term where applicable) were smoothly tapered off 
TABLE I. Room temperature simulations of $\mathrm{Cl}^{-}$and $\mathrm{Fe}^{2+}$ in water with either one of three water models and using either a cubic minimum image cutoff or Ewald summation.

\begin{tabular}{|c|c|c|c|c|c|}
\hline & & \multicolumn{2}{|c|}{$\mathrm{Cl}^{-}$} & \multicolumn{2}{|c|}{$\mathrm{Fe}^{2+}$} \\
\hline & & $\begin{array}{c}E_{\text {ion-wat }} \\
\left(\mathrm{kJ} \mathrm{mol}^{-1}\right)\end{array}$ & $\begin{array}{c}\Delta E \\
\left(\mathrm{~kJ} \mathrm{~mol}^{-1}\right)\end{array}$ & $\underset{\left(\mathrm{kJ} \mathrm{mol}^{-1}\right)}{E_{\text {ion-wat }}}$ & $\begin{array}{c}\Delta E \\
\left(\mathrm{~kJ} \mathrm{~mol}^{-1}\right)\end{array}$ \\
\hline $\begin{array}{l}\text { SPC } \\
\text { SPC }\end{array}$ & $\begin{array}{l}\text { Cutoff } \\
\text { Ewald }\end{array}$ & $\begin{array}{l}-606 \pm 11 \\
-543 \pm 8\end{array}$ & 63 & $\begin{array}{l}-2672 \pm 1 \\
-1975 \pm 3\end{array}$ & 697 \\
\hline $\begin{array}{l}\text { TIP4P } \\
\text { TIP4P }\end{array}$ & $\begin{array}{l}\text { Cutoff } \\
\text { Ewald }\end{array}$ & $\begin{array}{l}-605 \pm 2 \\
-549 \pm 2\end{array}$ & 56 & $\begin{array}{l}-2515 \pm 15 \\
-1792 \pm 6\end{array}$ & 723 \\
\hline $\begin{array}{l}\text { MCY } \\
\text { MCY }\end{array}$ & $\begin{array}{l}\text { Cutoff } \\
\text { Ewald }\end{array}$ & $\begin{array}{l}-672 \pm 9 \\
-640 \pm 4\end{array}$ & 32 & $\begin{array}{r}-2640 \pm 12 \\
1859 \pm 30\end{array}$ & 781 \\
\hline
\end{tabular}

over a range of $0.2 \AA$ with the product of three separate spline functions that were functions of the pair distances $|\Delta x|,|\Delta y|$, and $|\Delta z|_{.}^{28,29}$

Standard rigid body molecular dynamics simulations at $25^{\circ} \mathrm{C}$, using the Verlet algorithm,SHAKE, and a time step of 3 fs, were carried out. ${ }^{2}$ After sufficient equilibration, constant temperature (NVT) runs of 10000 steps duration with velocity resampling every 100 steps, or (for the data in Table II) constant energy (NVE) runs of 100000 steps duration were performed. Error bars were obtained from subaverages over five consecutive segments of a given simulation.

\section{RESULTS}

Table I shows the calculated ion-solvent interaction energies and hydration energies of $\mathrm{Cl}^{-}$and $\mathrm{Fe}^{2+}$ at $25^{\circ} \mathrm{C}$, using either a cubic (box sized) cutoff or Ewald summation. As the extrinsic potential is proportional to the quadrupole moment of the unit cell, it is conceivable that the importance of the surface effects could ultimately depend on the size of the molecular quadrupole moment. We therefore show all results for three different water models-SPC, TIP4P, and MCY that have very similar dipole moments, but rather different quadrupole moments. ${ }^{30}$

From the data for $\mathrm{CI}^{-}$it can then be seen that the ionwater potential energy differs between Ewald and cutoff conditions by about $30-60 \mathrm{~kJ} \mathrm{~mol}^{-1}$, depending on the water model. Qualitatively the same observations are made for $\mathrm{Fe}^{2+}$, but in this case the ion-water energy varies by as much as about $700-800 \mathrm{~kJ} \mathrm{~mol}^{-1}$. The cutoff data shown refer to a cubic or "minimum image" cutoff, but similar energy values are also obtained under spherical cutoff conditions. ${ }^{31}$

This appreciable difference between cutoff and Ewald conditions has not been mentioned in the literature, even though the same potential functions are often used by different investigators with different kinds of boundary conditions. Indeed, it is striking that numerous simulation reports of ionic solutions (or of solutions with an excess electronic quantum solute) omit any quote of thermodynamic data and particularly solute-solvent energies.

Table II shows the calculated ion-solvent interaction energies and hydration energies for $\mathrm{Cl}^{-}$in TIP4P and for $\mathrm{Fe}^{2+}$ in SPC water also under the two other types of boundary conditions. In summary, we have (1) pure minimum image cutoff conditions, (2) Ewald summation in presence of a "polarization correction,"(3) minimum image cutoff conditions in presence of a "depolarization" correction, and (4) pure Ewald summation. While the extrinsic potential is present in methods 1 and 2, it is absent in methods 3 and 4 . If the effects of periodic replication were negligible relative to the surface polarization effects, method 1 (nonreplicated system) and method 2 (replicated system) should yield the same result. Analogously, the results from methods 3 and 4 should be the same.

From the data in Table II it can be scen that these identities are approximately fulfilled for both $\mathrm{CI}^{-}$and $\mathrm{Fe}^{2+}$. Thus the Ewald value can he well predicted by a cutoff simulation with depolarization correction, just as the cutoff value can also be recovered from an Ewald simulation with polarization correction. Clearly, polarization and depolarization correction are completely "symmetrical."

Finally, it can be shown that the extrinsic potential can even be "retroactively" eliminated (or introduced). For example, if a cutoff simulation of $\mathrm{Fe}^{2+}$ in water (with a "true" ion-solvent energy of $-2672 \mathrm{~kJ} \mathrm{~mol}^{-1}$ ) is subsequently analyzed using Ewald summation, an ion-solvent energy of $-2097 \mathrm{~kJ} \mathrm{~mol}^{-1}$ is found. (Essentially the same result is obtained if a depolarization correction is retroactively applied to the cutoff simulation.) This number is not too different from the energy values of -1975 and $-1978 \mathrm{~kJ} \mathrm{~mol}^{-1}$ (Table II) that are obtained in a genuine Ewald simulation and in a cutoff simulation with "instantaneous" depolarization correction, respectively. Hence, the fact that the solvent structure (as represented, for example, by the dielectric properties) is rather different in the presence or absence of the

TABLE II. Room temperature simulations of $\mathrm{Cl}^{-}$in TIP4P water and $\mathrm{Fe}^{2+}$ in SPC water under various types of boundary conditions.

\begin{tabular}{lccc}
\hline & $\mathrm{Cl}^{-}$in TIP4P & & $\mathrm{Fe}^{2+}$ in SPC \\
\cline { 2 - 2 } & $\begin{array}{c}E_{\text {ion-wat }} \\
\left(\mathrm{kJ} \mathrm{mol}^{-1}\right)\end{array}$ & & $\begin{array}{c}E_{\text {ion-wat }} \\
\left(\mathrm{kJ} \mathrm{mol}^{-1}\right)\end{array}$ \\
\hline Cutoff & $-605 \pm 2$ & & $-2672 \pm 1$ \\
Ewald +extrinsic potential & $-609 \pm 6$ & & $-2695 \pm 15$ \\
Cutoff-extrinsic potential & $-547 \pm 3$ & & $-1978 \pm 9$ \\
Ewald & $-549 \pm 2$ & & $-1975 \pm 3$ \\
\hline \hline
\end{tabular}


extrinsic potential, ${ }^{31}$ is only of secondary importance. For the ion-solvent energy it is predominantly the sheer magnitude of the surface term that matters, rather than detailed structural correlations.

The different solvent structure does become important for other solute properties, as elucidated in a more comprehensive study of the properties of aqueous ionic solutions under different kinds of boundary conditions. ${ }^{31}$

\section{CONCLUSIONS}

That the electrostatic potential in a periodically replicated system is not a well-defined quantity was pointed out by Von Laue no less than 64 years ago. ${ }^{32}$ As mentioned in $\mathrm{Sec}$. I, the phenomenon is indeed regularly encountered in a solid-state physics context, where the crystalline microscopic order ordinarily gets reproduced on a macroscopic scale. In the present study, we have elaborated on how the same problem arises in simulations of disordered systems. In the case of minimum image boundary conditions, it is that the system is not completely disordered on the length scale of the truncation unit. In the case of lattice summation it is that the same order gets retained over long distances by the periodic replication. Thus we are dealing with a peculiar small system effect, arising in conjunction with typical condensed phase boundary conditions.

The difference is the extrinsic potential which, under fixed-cell conditions, is related to the dipolar and the quadrupolar moment of the unit cell. Under centered-cell conditions it is only related to the quadrupolar moment. Neither the dipole moment nor the quadrupole moment will generally vanish in a disordered system. Presence of quadrupole moments alone is sufficient to lead to absolute shifts of singleion solvation energies, Eqs. (5) or (6). Presence of dipole moments will also indirectly affect solvation energies by truly changing the Hamiltonian, Eqs. (9) and (13), and thus the structure (and dynamics) of the simulated system.

Now it is true that what, strictly speaking, should be compared with the experiment is only the solvation energies of ion pairs. In that case the absolute energy shifts for anion and cation will cancel and in that sense there is at least no problem with the described direct surface effects, Eqs. (5) or (6). Still. what is normally tabulated is single-ion solvation energies, and the convention is to define the latter relative to a proton that is taken from vacuum to a water sample in the thermodynamic limit, i.e., in the absence of extrinsic potentials. Thus experimental solvation energies, but not necessarily simulation values, exclude surface effects and it is at this point that even the absolute shifts in energy become important.

Then which of the ion-solvent energies quoted are the real values for $\mathrm{Cl}^{-}$and $\mathrm{Fe}^{2+}$ in water? One might say the cutoff energies, in that the potential functions usually used in computer simulations are constructed to give physically meaningful energies and forces under molecular minimum image conditions with surrounding vacuum. In a typical fitting procedure, the potential parameters are obtained by fitting dimers or other small clusters against experimental thermodynamic data, etc. Naturally, Ewald energies are to be preferred if the parametrization is carried out under Ewald conditions. Thus information about how the parametrization has been carried out should be an integral part of the characterization of a potential function.

Nevertheless, it is still true that Ewald summation is, for principal reasons, the preferred type of boundary condition. It has in fact been empirically found, and is even more often conjectured, that Ewald summation leads to more desirable results, in particular for the calculation of dielectric properties. This can now be rationalized: Ewald summation is an elegant tool to circumvent the problems associated with the surface charge distribution. This is different from the solidstate physics situation where Ewald summation provides unique values of the potential, but at the cost of introducing a potentially unphysical boundary condition, namely surrounding metal, that can potentially lead to wrong results for the energy bands of the solid. In the disordered system case, however, the effect of Ewald summation is always going to be to eliminate the disturbing extrinsic potential.

From our results it is also clear that depolarization correction is a very simple and computationally expedient alternative for obtaining simulation results similar to Ewald summation. It is now striking that the surface term, Eq. (9), has exactly the same structure as a reaction field Hamiltonian. The conventional rationalization of the reaction field method is that water is a medium of very high dielectric constant and thus the surrounding dielectric continuum figures as a longrange correction to the energies calculated with a short-range cutoff. It is sometimes said that the Ewald method is justified by the fact that it approximates the reaction field, at least in a medium of high dielectric constant. ${ }^{33}$ The arguments put forth here appear to indicate that one might also take the viewpoint that the Ewald method is always to be preferred, even for media of low dielectric constant, in that it eliminates the extrinsic potential. Conversely, the reaction field method, if implemented with an infinite dielectric constant, is justified as accomplishing the same.

In summary, we conclude that while Ewald summation is generally the preferred type of boundary condition, ionsolvent energies calculated with conventional cutoff schemes are presumably more appropriate because of the genesis of typical potential functions.

\section{ACKNOWLEDGMENTS}

We thank Professor Hans C. Andersen for a helpful discussion. Acknowledgment is made to the Donors of The Petroleum Research Fund, administered by the American Chemical Society, for partial support of this research (Grant No. 25299-G6). The computations were performed with resources provided by a NSF Instrumentation Grant (No. CHE8917309 ) to the Department of Chemistry of the University of Michigan.

\section{APPENDIX}

The implementation of periodic boundary conditions in a condensed phase simulation requires a number of specifications, many of which are often only implicitly made. We find the following classification convenient. 
(a) Choice of lattice of unit cells. Even though a spacefilling three-dimensional lattice of unit cells can be realized with any one of the 14 Bravais lattices, only the simple cubic lattice, the body-centered cubic lattice, and the face-centered cubic lattice are popular. The corresponding Wigner-Seitz cells (=unit cells) are the simple cube, the truncated octahedron, and the rhombic dodecahedron, respectively. ${ }^{2}$

(b) Choice of unit cell surface. It is possible to lower the unit cell symmetry by allowing for distortions (or, generally, any nonplanar shape) of the faces of the cell; because of the periodicity constraint, the distortions of any two opposing faces will have to be identical. While this is normally not done in any ad hoc fashion, this is still regularly encountered in molecular liquids if these are simulated under the usual "molecular minimum image" conditions (to be discussed below). The latter provide for a constantly changing "surface corrugation" of the interaction cell (see below) which is most easily also applied to the unit cell itself.

(c) Periodic versus toroidal boundary conditions. Next, we must distinguish between the lattice sums evaluated in a truly replicated system (periodic boundary conditions in the literal sense), and the toroidal boundary conditions ${ }^{34}$ that are obtained by only including the nearest images in the evaluation of potentials and forces.

(d) Fixed-cell mode versus centered-cell mode. The energy can be evaluated in two fundamentally different ways. One possibility is to define a unit cell with a distinct origin, subject to periodic replication. The energy is then obtained from all pair distances inside the array of unit cells, under division by the number of cells. This is the exact analog of the usual energy expression for a cluster. The direct lattice sums analyzed by De Leeuw et al. ${ }^{12}$ and Olives ${ }^{22}$ are examples of the fixed-cell mode.

The other possibility implies that the minimum image convention is somehow invoked, regardless of the number of images actually included in the energy evaluation. Most commonly the centered-cell mode will be implemented in conjunction with toroidal boundary conditions, thereby providing for "the" minimum image convention. If infinite replication of the unit cell is combined with the centered-cell mode, we obtain "centered-cell lattice sums" where each particle is at the center of its own periodically replicated array of unit cells. This is the generalization of the usual minimum image convention to the case of more than one image.

The importance of the distinction between fixed-cell and centered-cell mode pertains to the fact that even though the standard mathematical treatments are based on the former, all dynamic simulation schemes automatically correspond to the latter (unless surface effects have been eliminated, e.g., via Ewald summation, in which case the distinction becomes irrelevant). That fixed-cell conditions would lead to frequent jumps of the total energy is easily seen if it is recalled that most particles are at different distances from any two opposing faces of the unit cell. Thus there is a discontinuity in the pair energy between any off-center particle and a surface particle whenever the latter "leaves" on one side of the unit cell while the corresponding image particle "re-enters" on the other side (in the dynamic implementation of periodic boundary conditions).

This problem can actually be fixed by completely removing the "dynamic aspect" of the periodic boundary conditions and simply keeping track of continuous particle trajectories. Naturally, this would lead to a gradual "dissolution" of the unit cell, just as in the simulation of a cluster under free boundary conditions. Since this does not appear to be very practical, only the centered-cell mode will allow for an energy-conserving algorithm.

The restriction to centered-cell conditions does not necessarily apply to Monte Carlo simulations since, in this case, the fixed-cell mode-induced energy jumps between configurations can conceivably be ignored in the sense that these simply get 'absorbed' by the stochastic algorithm.

(e) Choice of size and surface of interaction cell. Besides the unit cell surface, we must also specify the size and the shape of the region that is actually seen by a particle during the evaluation of the pair interactions. Using finite lattice sums, this refers to the shape and the volume of the spatial region within which the unit cell is periodically replicated; in the case of infinite lattice sums, we must specify the shape of the expanding surface of unit cells as more and more "shells" are added to the system. In either case, the most common shape is that of a sphere (e.g., the normal spherical cutoff), but other geometries, such as a cubic shape (for example, for minimum image truncation), are also possible.

$(f)$ Interactions by sites versus interactions by molecules. In most models of molecular liquids the electrostatic potential is not represented through point dipoles, or generally point multipoles, but rather through distributed partial charges whose positions mostly coincide with the nuclear positions. We then need to decide if the specific conditions for the evaluation of the interactions are to be applied site by site, or rather molecule by molecule. ${ }^{3(g)}$ In short we have to distinguish between site minimum image shifting and molecular minimum image shifting. By alluding to the unambigeous energy expression for clusters, it is clear that the latter set of conditions will normally provide for the physically desired case. Hence, the surface of the interaction cell will generally be "corrugated" as we are requiring that only molecules as a whole, with some interaction centers potentially "outside" of the mathematically ideal cell, be assigned to a given side.

It is only in the case of Ewald summation that this distinction becomes irrelevant as the potential is strictly periodic.

(g) Surroundings. Finally, we have to distinguish between the standard practice of surrounding the interaction cell by vacuum and that where each interaction cell is immersed in a medium of some dielectric constant $\epsilon$. If the latter is infinite $(\epsilon=\infty)$ we have the conducting or tin foil boundary conditions that are implicitly present in simulations that use Ewald simulation. Explicitly, this kind of boundary condition is also realized in simulations that combine cutoff conditions with a reaction field technique, in which case the dielectric constant $\epsilon$ of the surrounding medium can be arbitrarily specified. 
'The Problem of Long Range Forces in the Computer Simulation of Condensed Media, edited by D. Ceperley, NRCC Proceedings No. 9 (Lawrence Berkeley Laboratory, 1980).

${ }^{2} \mathrm{M}$. P. Allen and D. J. Tildesley, Computer Simulation of Liquids (Clarendon, Oxford, 1987), and references therein.

${ }^{3}$ Recent work on boundary conditions in water and aqueous solutions includes: (a) M. Prevost, D. van Belle, G. Lippens, and S. Wodak, Mol. Phys. 71, 587 (1990); (b) M. Belhadj. H. E. Alper, and R. M. Levy, Chem. Phys. Lett. 179, 13 (1991); (c) P. E. Smith and B. M. Pettitt, J. Chem. Phys. 95, 8430 (1991); (d) J. S. Bader and D. Chandler, J. Phys. Chem. 96, 6423 (1992); (e) H. Schreiber and O. Steinhauser, Chem. Phys. 168, 75 (1992): (f) F. S. Lee and A. Warshel, J. Chem. Phys. 97, 3100 (1992); (g) K. Tasaki, S. McDonald, and J. W. Brady, J. Comp. Chem. 14, 278 (1993); (h) H. E. Alper, D. Bassolino, and T. R. Stouch, J. Chem. Phys. 98, 9798 (1993).

${ }^{4}$ See, for example, N. W. Ashcroft, and N. D. Mermin, Solid State Physics (Saunders College, Philadelphia, 1976), Chaps. 18, 20.

${ }^{5}$ A. Redlack and J. Grindlay, Can. J. Phys. S0, 2815 (1972); J. Phys. Chem. Solids 36, 73 (1975).

'S. N. Stewart, J. Comp. Phys. 29, 127 (1978).

${ }^{7}$ R. N. Euwema and G. T. Surratt, J. Phys. Chem. Solids 36, 67 (1975).

${ }^{8}$ S. Kuwajima and A. Warshel, J. Chem. Phys. 89, 3751 (1988).

${ }^{9}$ E. R. Smith, Proc. R. Soc. London Ser. A 375, 475 (1981).

${ }^{10} \mathrm{H}$. M. Evjen, Phys. Rev. 39, 675 (1932).

"P. P. Ewald, Ann. Phys. 64, 253 (1921). See also M. P. Tosi, in Solid State Physics, Vol. 10, edited by F. Seitz and D. Turnbull (Academic, New York, 1964).

${ }^{12}$ S. W. De Leeuw, J. W. Perram, and E. R. Smith, Proc. R. Soc. London Ser. A 373, 27 (1980).

1.3. B. Felderhof, Physica 101A, 275 (1980).

${ }^{14}$ B. J. Alder and E. L. Pollock, Annu. Rev. Phys. Chem. 32, 311 (1981).

15. M. Neumann, Mol. Phys. 57, 97 (1986).
${ }^{16} \mathrm{~S}$. W. De Leeuw, P. W. Perram, and E. R. Smith, Annu. Rev. Phys. Chem. 37, 245 (1986)

${ }^{17}$ H. L. Friedman, A Course in Statistical Mechanics (Prentice Hall, Englewood Cliffs, NJ, 1985), Chap. 5.

${ }^{18} \mathrm{~J}$. P. Hansen and I. R. McDonald, Theory of Simple Liquids, 2nd ed. (Academic, London, 1986), Chap. 12.

${ }^{19}$ M. J. Sangster and M. Dixon, Adv. Phys. 25, 247 (1976).

${ }^{20}$ J. P. Valleau and S. G. Whittington, in Statistical Mechanics, Part A, edited by B. J. Berne (Plenum, New York, 1977).

${ }^{21}$ By expanding in spherical harmonics, Redlack and Grindlay (Ref. 5) also arrive at a completely general, but only approximate expression for the extrinsic potential within a region of arbitrary (nonellipsoidal) shape.

${ }^{22}$ J. Olives, J. Phys. Lett. (Paris) 46, L1143 (1985).

${ }^{23}$ H. J. C. Berendsen, J. P. M. Postma, W. F. van Gunsteren, and J. Hermans, in Intermolecular Forces, edited by B. Pullman (Reidel, Dordrecht, 1981).

${ }^{24}$ W. L. Jorgensen, J. Chandrasekhar, J. D. Madura, R. W. Impey, and M. L. Klein, J. Chem. Phys. 79, 926 (1983).

${ }^{25}$ O. Matsuoka, E. Clementi, and M. Yoshimine, J. Chem. Phys. 64, 1351 (1976).

${ }^{26} \mathrm{~J}$. Chandrasekhar, D. C. Spellmeyer, and W. I. Jorgensen, J. Am. Chem. Soc. 106, 903 (1984).

${ }^{27}$ L. A. Curtiss, J. W. Halley, J. Hautman, and A. Rahman, J. Chem. Phys. 86, 2319 (1987).

${ }^{28}$ D. J. Adams, E. M. Adams, and G. J. Hills, Mol. Phys. 38, 387 (1979).

${ }^{29}$ O. Steinhauser, Mol. Phys. 45, 335 (1982).

${ }^{30}$ J. R. Reimers, R. O. Watts, and M. L. Klein, Chem. Phys. 64, 95 (1982); K. Watanabe and M. L. Klein, ibid. 131, 157 (1989).

${ }^{31}$ J. E. Roberts and J. Schnitker (submitted).

${ }^{32}$ M. Von Laue, Sitzungsber. Preuss. Akad. Wiss. Phys.-Math. KI. 1930, 26.

${ }^{33}$ See, for example, J. A. Barker, in Ref. 1.

${ }^{34}$ M. Neumann and O. Steinhauser, Mol. Phys. 39, 437 (1980). 